\title{
Fractal dimension in the evaluation of different treatments of muscular injury in rats
}

\author{
Thiago Alves Garcia a,*, Guilherme Akio Tamura Ozaki ${ }^{a}$, Robson Chacon Castoldi ${ }^{\mathrm{a}}$, \\ Tatiana Emy Koike ${ }^{\mathrm{b}}$, Regina Celi Trindade Camargo ${ }^{\mathrm{c}}$, José Carlos Silva Camargo Filho ${ }^{\mathrm{c}}$ \\ ${ }^{\text {a } U n i v e r s i d a d e ~ E s t a d u a l ~ d e ~ C a m p i n a s ~(U N I C A M P), ~ P r o g r a m a ~ d e ~ P o ́ s-g r a d u a c ̧ a ̃ o ~ e m ~ C i e ̂ n c i a s ~ d a ~ C i r u r g i a, ~ C a m p i n a s, ~ S P, ~ B r a z i l ~}$ \\ ${ }^{\mathrm{b}}$ Universidade de São Paulo (USP), Programa de Pós-graduação em Ciências Morfofuncionais, São Paulo, SP, Brazil \\ ${ }^{\mathrm{c}}$ Universidade Estadual Paulista (UNESP), Departamento de Fisioterapia, Presidente Prudente, SP, Brazil
}

\section{A R T I C L E I N F O}

\section{Keywords:}

Fractal dimension

Histology

Collagen

Platelet rich plasma

Low level laser therapy

\begin{abstract}
A B S T R A C T
Objectives: To evaluate alterations from different therapies in muscular injury using the Fractal Dimension (FD) method.

Methods: 35 animals were allocated in Control Group (C), Injury Control Group (IC), Injury Low Level Laser Therapy Group (ILT), Injury Platelet Rich Plasma Group (IP), and Injury LLLT and PRP Group (ILP). The animals suffered a stretch injury in gastrocnemius muscle and after that IP and ILP groups received PRP application. The ILT and ILP groups received daily LLLT applications for seven days. After seven days the animals were euthanized and the gastrocnemius muscle removed and frozen. The muscles were stained with Hematoxylin and Eosin (HE) and Picrosirius Red, for observation of the morphology of the injury and semi-quantitative and quantitative analysis through the Fractal Dimension (FD) method.

Results: In the qualitative and semi-quantitative analysis, in relation to IC group, the ILT presented a reduction in rounded fibers and the IP in angular fibers. The ILP group demonstrated a reduction in both polymorphic fibers and inflammatory infiltrate. The FD of the muscles stained with HE was higher in the groups that suffered the injury when compared to the $C$ group ( $p<0.05$ ); the FD of the collagen demonstrated no statistical difference between the groups.

Conclusion: Both treatments were able to accelerate injury repair, and the association of both presented better results than the isolated applications. However, the FD method showed no sensitivity to differentiate the treatments, either in the histological aspects or the injury in collagen.
\end{abstract}

\section{Introduction}

Muscle tissue injuries are a common problem in every population. These can be caused by work, sports, or accidents and due to their frequency more efficient treatments are required (Edouard et al., 2016; Astur et al., 2014). Among the principal treatments for these lesions are low intensity laser therapy (LLLT) and platelet rich plasma.

LLLT decreases inflammation and oxidative stress in muscle injury, in addition to increasing the production of growth factors responsible for angiogenesis and myogenesis (Silveira et al., 2016; Adabbo et al., 2016). On the other hand, PRP contains in its composition a concentration of anucleated cells that release cytokines (TGF-1 $\beta$ ), myogenic factors (MyoD1, MYF5), and growth factors (IGF-IEb) responsible for activating muscle repair and regeneration pathways during the process of inflammation (Dimauro et al., 2014; Li et al., 2016).

Evaluating the degree of injury and efficiency of treatments with precision has presented a challenge, since the majority of histological analysis methods are qualitative, and depend on the degree of instruction of the evaluator. In this context, fractal dimension (FD) presents itself as a viable tool to evaluate the cellular morphology with the minimum of interference from the evaluator.

FD is a mathematical measure that quantifies the complexity of a figure of irregular (non-Euclidean) geometry. In this way it fits as an ideal and reproducible tool to deal with the complexity and irregularity of muscle cells.

Analysis of fractal objects is based on the relationship between the resolution and scale at which the object is measured. The results can be expressed quantitatively by the equation:

\footnotetext{
* Corresponding author at: Laboratório da Plasticidade muscular, Faculty of Science and Technology - UNESP/FCT, Rua Roberto Simonsen, 305 - Jardim das Rosas Presidente Prudente, 19060-900, SP, Brazil.

E-mail address: t207409@dac.unicamp.br (T.A. Garcia).
} 
$\mathrm{FD}=(\log \mathrm{Nr} / \log \mathrm{r}-1)$

In this formula "Nr" is the number of elements necessary to overlap or fill the original object, " $\mathrm{r}$ " represents the scale applied to the object, and "FD" is the dimension of the structure or object.

The FD can be calculated in several ways, the "box-counting method" being the most common in histology. In this method the image is covered with progressively smaller squares of " $\mathrm{r}$ " sides and thus " $\mathrm{Nr}$ " is the number of squares of side " $r$ " needed to cover the image, for each chosen size. The FD will then be obtained by the slope of the regression line of the two log-values, that is, the size of the " $r$ " side and the number of squares "Nr"(7).

FD has been used in many ways, be it to analyze tissues such as neurons, retina, and bronchi, or detect carcinomas and level of rejection of cardiac cells after heart transplantation (Moreira et al., 2011; Ristanović et al., 2014; de Melo de Mendonça et al., 2007; Gupta et al., 2014; Lee et al., 2014). However information on its use for measurement of different types of treatment for muscle injury is still scarce. This technique could present a more sensitive and effective method to evaluate the degree of injury and consequently the degree of muscle, independent of the evaluator.

Therefore the objective of the present study is to verify the differences in different treatment protocols in the muscle injury of rats using the fractal dimension method.

\section{Methods}

Thirty-five male Wistar (Rattus novergicus) rats (aged 150 days) were used, acquired from the central Bioterium of Paulista State University (UNESP), Botucatu-SP Campus (Brazil). They were maintained at the bioterium of the Histology and Histochemistry Laboratory at the Faculty of Science and Technology, Presidente Prudente (FCT/ UNESP), in collective cages (polyethylene), at a controlled temperature $\left(22 \pm 2{ }^{\circ} \mathrm{C}\right)$, humidity $(50 \pm 10 \%)$, and 12 -hour light/dark cycle with access to food (standard laboratory chow) and water ad libitum.

All procedures were approved by the ethics committee for animal use from FCT/UNESP, Presidente Prudente (SP, Brazil) campus, protocol no. $01 / 2013$.

\subsection{Experimental groups}

The animals were randomly divided into five groups: Control (C); Control Injury (IC); Injury and Low Level Laser Therapy (ILT); Injury and Platelet Rich Plasma (IP); Injury with both LLLT and PRP (ILP). The animals in group $C$ remained in the bioterium and were euthanized paired with the other groups. The animals in groups IC, ILT, IP, and ILP suffered the muscle injury and were euthanized seven days after injury. The ILT group animals received laser application daily for seven days. The IP animals received PRP application immediately after the injury. The ILP group received application of both protocols mentioned above.

\subsection{PRP preparation}

The animals of the IP and ILP groups underwent cardiac puncture for preparation of PRP and were then submitted to the muscle injury protocol. Blood collection was performed through cardiac puncture in the animals of the IP and ILP groups. The animals were submitted to anesthesia by intraperitoneal administration of ketamine $(70 \mathrm{mg} / \mathrm{kg}$ ) and xylazine $(15 \mathrm{mg} / \mathrm{kg})$, and after confirmation of anesthesia a cardiac puncture was performed using a $0.2 \mathrm{ml}$ disposable syringe containing sodium citrate at $10 \%, 4 \mathrm{ml}$ of blood was obtained from each animal. Immediately after the puncture, saline solution was injected to restore blood volume (Li et al., 2012).

The collected blood was centrifuged at $200 \mathrm{~g}$ for $15 \mathrm{~min}$, splitting the sample into three parts: red bottom fraction, composed primarily of red blood cells; intermediate yellow-straw fraction (buffy coat), with the serum component; and the top fraction, composed of the blood plasma. The top fractions were pipetted, including the buffy coat, and the pipetted contents were centrifuged again at $500 \mathrm{~g}$ for $10 \mathrm{~min}$. Next, $0.2 \mathrm{ml}$ of the bottom content PRP was pipetted.

Blood and PRP samples were analyzed in the laboratory of the Veterinary Hospital of the Universidade do Oeste Paulista (UNOESTE) by means of an automatic blood cell analyzer (pocH 100iy Diff, Sysmex ${ }^{\circledast}$ ). The analysis was performed on two blood samples and three PRP samples, for confirmation of platelets.

\subsection{Muscle injury protocol}

In the IP and ILP groups the muscle injury was performed immediately after cardiac puncture, avoiding the application of new doses of anesthesia. In the IC and ILT groups, the animals received an intraperitoneal injection of xylazine and ketamine, as described above. After confirmation of anesthesia, each animal was placed on the damage inductor equipment, in a supine position, with the hip in slight flexion, knee extension, and ankle in plantar flexion, the right leg attached to the machine with adhesive tape (duct tape). After positioning the animal in the equipment, two electrodes were placed on the paw, on the calcaneal tendon and popliteal fossa of the animal, respectively. Electrical stimulation was applied suddenly to the positioned animal until full contraction of the lower limb in plantarflexion. Immediately afterwards, the equipment was fired, which promoted abrupt dorsiflexion of the lower limb while it was stimulated, the electrical current was stopped immediately after dorsiflexion. The dorsiflexion stimulation caused by the equipment and interruption of the current, in total, took an average of $2 \mathrm{~s}$ to complete. This procedure was repeated until totaling 10 series, with a $30 \mathrm{~s}$ interval between applications. In each series $2.25 \mathrm{~J}$ was released, totaling $22.5 \mathrm{~J}$ of energy applied to the muscle injury. This protocol was adapted from Pachioni et al. (2009).

\subsection{PRP application}

In animals of the IP and ILP groups, $100 \mu \mathrm{l}$ of PRP was injected using a sterile syringe. The syringe with the needle was placed on the injured limb in the distal third of the tibia in order to be applied in the belly of the gastrocnemius muscle. The application of PRP was performed within six hours of the muscle injury protocol and withdrawal of blood (Hammond et al., 2009).

\subsection{Low level laser therapy application}

LLLT was applied by means of diode laser equipment (Coherent, Laser Cube) and previously calibrated with a wavelength of $637 \mathrm{~nm}$, power of $25 \mathrm{~mW}$, beam diameter of $1 \mathrm{~mm}$, and continuing emission for $10 \mathrm{~s}$. Therefore, the dosage was $0.25 \mathrm{~J} / 0.00785 \mathrm{~cm}$ (Astur et al., 2014) or $31.85 \mathrm{~J} / \mathrm{cm}$ (Astur et al., 2014). The laser was applied to a single point, daily, starting on the day of injury until the completion of seven applications (Iyomasa et al., 2009; Luo et al., 2013). The laser was applied at the same point as the PRP injection, both to the muscle belly region.

\subsection{Histological process and analysis}

After the experimental period, the animals were euthanized with an overdose of anesthetic xylazine and ketamine. Next, the right gastrocnemius muscle was removed from each animal and immersed in nhexane; cooled in liquid nitrogen, using the freezing method for nonfixed tissues (JCSC et al., 2011); and stored in an ultra-low temperature freezer, Coldlab CL580-80 V, at $-75^{\circ} \mathrm{C}$. The slides for histological analysis were stained with the hematoxylin and eosin (HE) and picrosirius methods (Castoldi et al., 2013).

Histological analysis was performed in a qualitative and semiquantitative way on the slides stained with HE, using a Nikon Eclipse 
$50 \mathrm{i}$ microscope. The qualitative analysis was based on the morphology of the muscle fibers, based on features such as the shape (polygonal, rounded, angular), connective tissue (endomysium and the perimysium), and inflammatory infiltrate fibers in a state of necrosis and phagocytosis. The semi-quantitative analysis of morphological characteristics was performed by the frequency of occurrence in each animal and classified according to intensity: absent (0), mild (Edouard et al., 2016), moderate (Astur et al., 2014), and intense (Silveira et al., 2016). This method was adapted from De Souza (De Souza et al., 2011). The picrosirius stained slides were photographed using a Leica DM 4000B polarized light microscope, linked to a Leica DFC500 camera, belonging to the Faculty of Dentistry, UNESP, Araçatuba - SP, Brazil. The images were obtained with a $10 \times$ objective lens.

For the fractal analysis of the images, the HE and picrosirius stained images were submitted to a binarization process, turning them black and white. At this point the HE images presented the nucleus of the cells black and the rest of the cell white. By means of this method we evaluated the inflammatory infiltrate from the inflammatory process triggered by muscle injury. The images of collagen (picrosirius), when observed using a polarized microscope, showed the collagen fibers in red, green, and yellow, and the rest of the cell as black. After the binarization process all collagen areas became black and the rest of the cell white. In this case the analysis was performed on the entire area of collagen, as the colors that differentiated the types of collagen had all been turned black (Fig. 1).

Subsequently the analysis of FD was performed using the boxcounting method. All procedures, including the preparation process, were performed using Image $\mathrm{J}$ software, available for free on the internet (http:// rsbweb.nih.gov/ij/). The sizes of the boxes used in the analysis were the default sizes of the software $(2,3,4,6,8,12,16,32$, and 64).

The graphic $\log / \log$ was made with the mean of the number of the box in each group and the size of the boxes, both were converted into $\log$.

\section{Statistical analysis}

The data were tested for normality using the Shapiro-Wilk test. As normal distribution was demonstrated, ANOVA one-way was used with the Tukey post-test to verify the differences between groups. For all analyzes the significance level was set at $5 \%$.

\section{Results}

The platelet quantity found in PRP was averaged at $4998.676 \times 10$ (Silveira et al., 2016) platelets/ $\mu \mathrm{L}$ of blood, a number four times larger than that found in the blood of the animal $(1068 \times 10$ (Silveira et al., 2016) platelets/ $\mu \mathrm{L}$ of blood).

\subsection{Histological analysis}

In qualitative analysis we found in group C, fibers with polygonal formats, and absence of inflammatory infiltrate. The perimysium and endomysium presented normal thickness. The groups submitted to the injury process (IC, ILT, IP, ILP), demonstrated a process of inflammation, necrosis, and phagocytosis in the muscle, as well as fibers with signs of stress such as polymorphic, rounded, and angular. There were no signs of alteration in the perimysium or endomysium of these groups (Fig. 2).

In the intervention groups, the inflammatory infiltrate was less extensive compared to the CI, moreover, these infiltrates were denser, presenting more cells in the area of the injury.

In the treated groups the muscle fibers demonstrated less signs of stress in comparison with the IC, these having a format closer to polygonal. Regarding the extracellular matrix, none of the treatments altered its thickness.

\subsection{Semi-quantitative analysis}

The data obtained by mean and standard deviation demonstrate in IC group the highest means of intensity among all groups. In the ILT
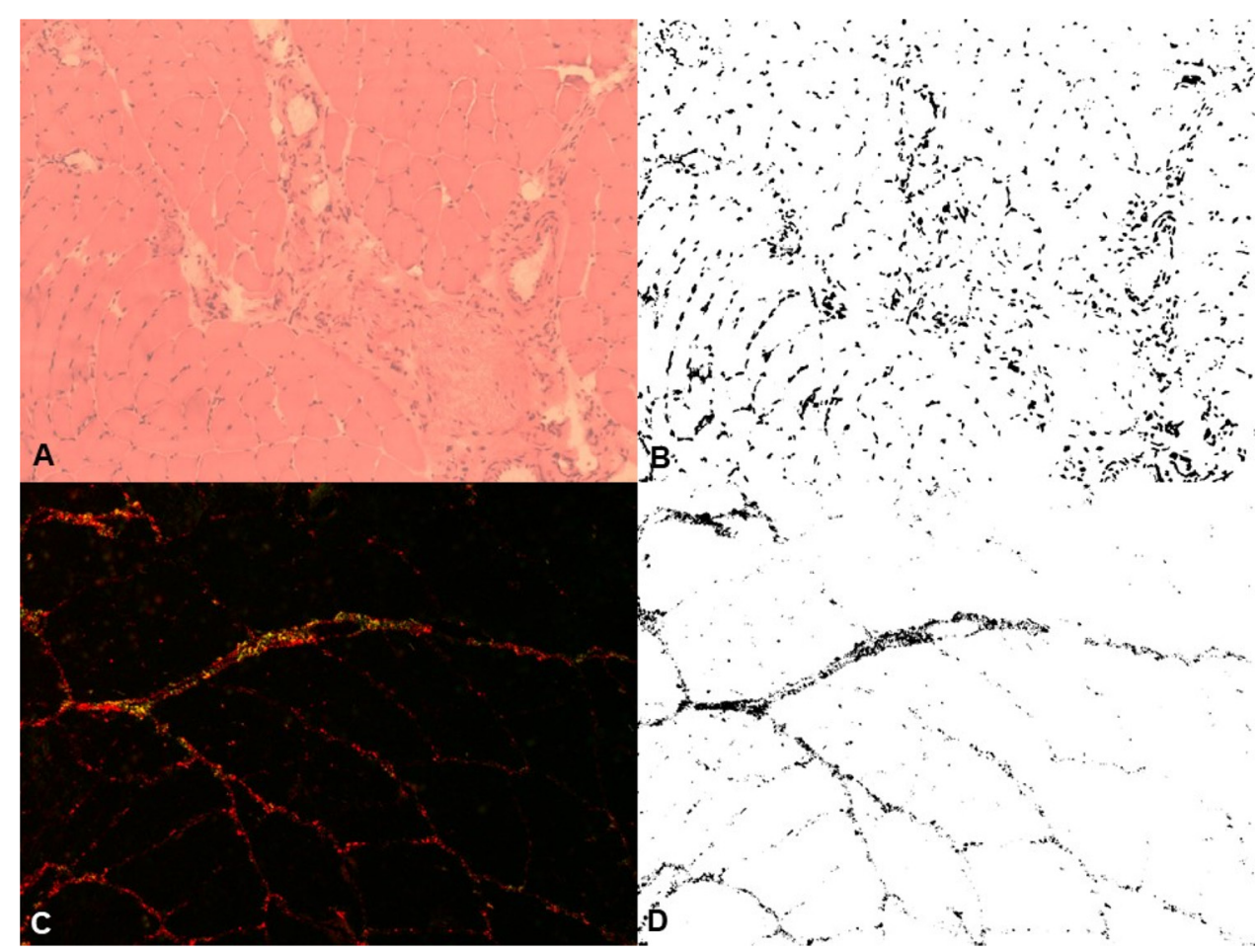

Fig. 1. The process of binarization necessary previous the Fractal Dimension analysis. A $=H E 200 \times$ magnification. $B=H E 200 \times$ magnification binarized. $\mathrm{C}=$ Picrosirius red $100 \times$ magnification. $\mathrm{D}=$ Picrosirius red $100 \times$ magnificantion binarized. 


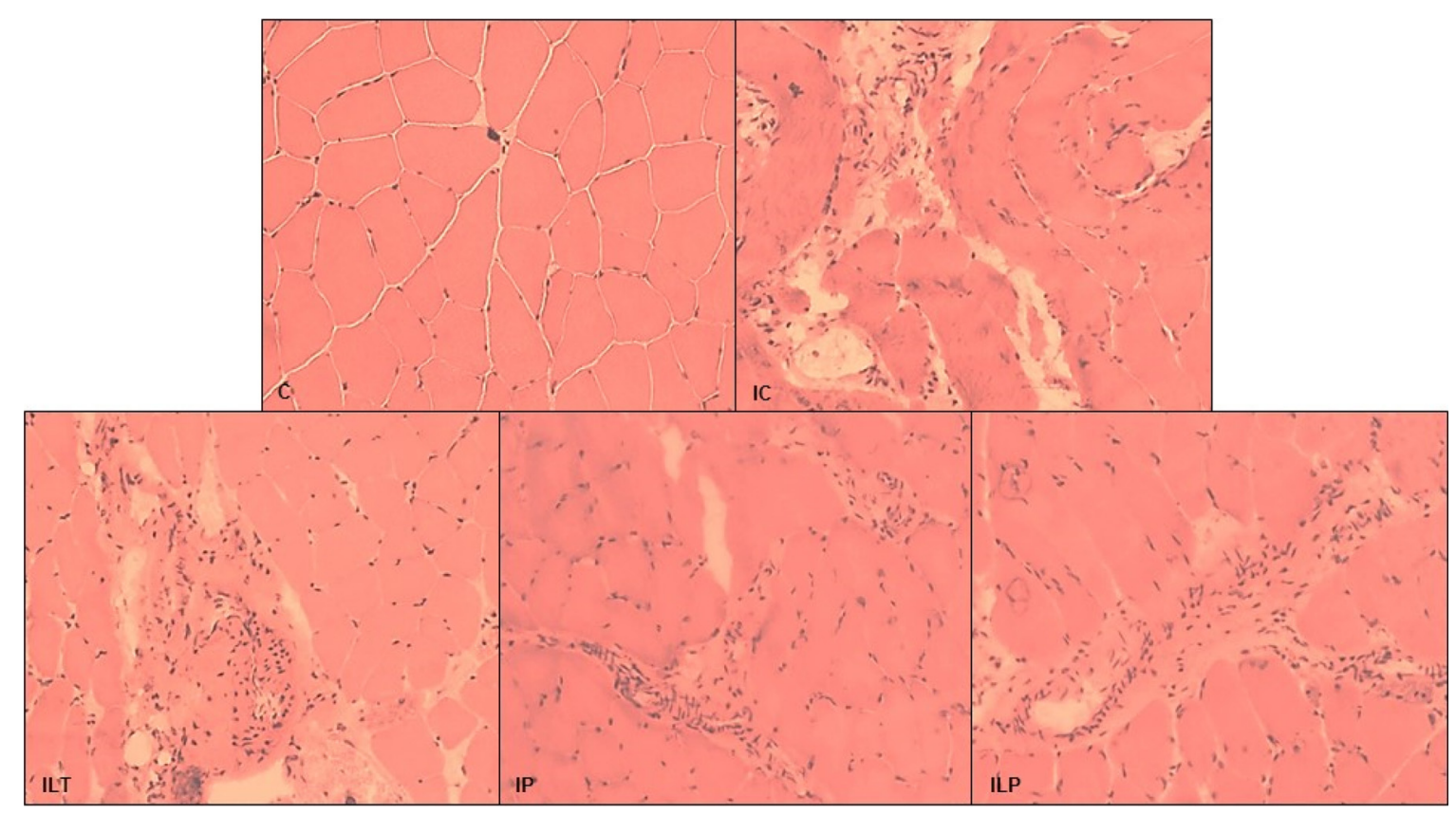

Fig. 2. Images of histological slides stained with HE, magnification $200 \times . \mathrm{C}=$ Control; IC $=$ Control Injury; ILT $=$ Injury Low Level Laser Therapy; IP $=$ Injury Platelet Rich Plasma; ILP = Injury LLLT and PRP.

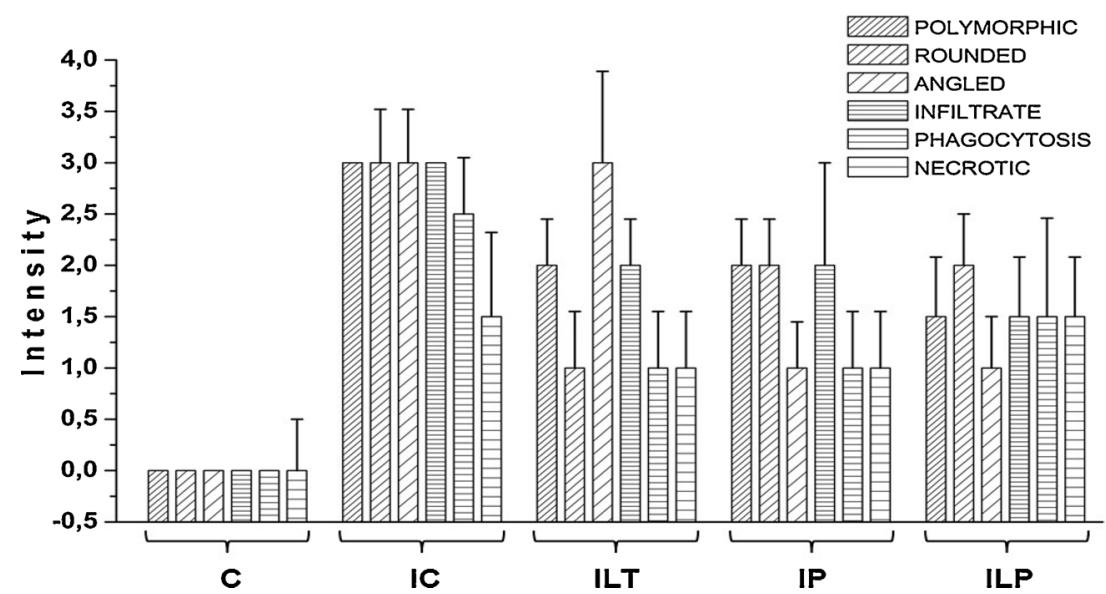

Fig. 3. Values of means and standard deviation of morphological characteristics. C = Control; IC = Control Injury; ILT = Injury Low Level Laser Therapy; IP = Injury Platelet Rich Plasma; ILP = Injury LLLT and PRP.

group, except for the angular fibers that presented values similar to those found in the IC group, the other values were below those in the injured groups. Both treatments reduced the morphological characteristics of the injury, especially the association of treatments, which presented the lowest values for polymorphic fibers and inflammatory infiltrate (Fig. 3).

\subsection{Fractal analysis}

The Fractal Dimension of the slides stained with HE, were calculated based on 6 images of group $C$ and 12 to 13 images from the other groups. They showed greater values in groups submitted to the injury process. This difference was statistically significant $(\mathrm{p}<0.05)$, with the group $\mathrm{C}$ being lower than the values of the other groups (IC, ILT, IP, ILP) (Fig. 4).

Collagen analyses were calculated with 6-14 images of each group. The groups presented similar values of mean and standard deviation, except for the IP group which demonstrated a greater standard deviation than the other groups. No statistical difference was found between groups (Fig. 5).

Below there are the graphs with the $\log / \log$ of the mean values found for each group analyzed in their respective analysis (Fig. 6).

\section{Discussion}

In the present study it was observed that FD was not able to identify the histological alterations promoted by the treatments with Low Intensity Laser Therapy, Platelet Rich Plasma, and the association of both in muscle tissue after injury.

Muscle tissue, when stained with Hematoxylin and Eosin (HE), allows observation of structures such as the cell edge, cytoplasm, nucleus, and extracellular matrix; after the binarization of this image only the nuclei are observed. When the FD method is applied to these images, the association of the shapes with the sizes of the nuclei is measured, and the values obtained represent the complexity of the image.

Regardless of the method of injury used, the pattern of regeneration occurs through the phases of destruction, repair, and remodeling. During the destruction and repair phases, the infiltration of cells such as 


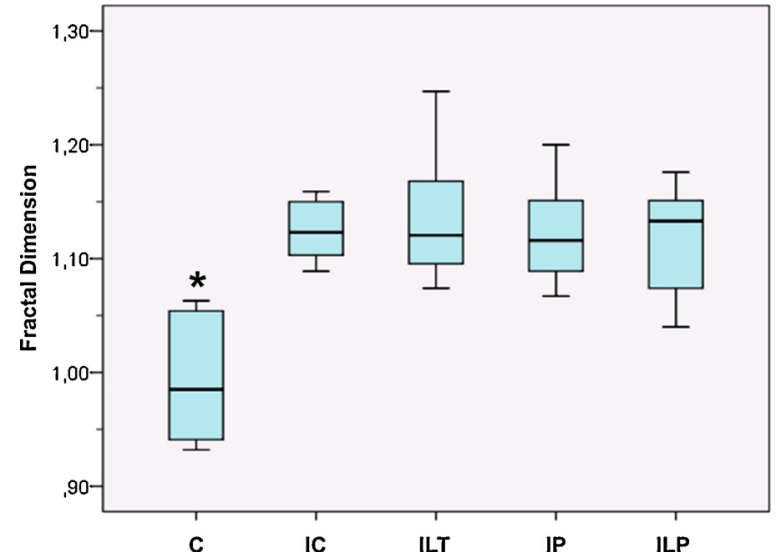

Fig. 4. Boxplot of fractal analysis of slides stained with Hematoxylin and Eosin. * Statistical difference compared to IC $(p<0,01)$, ILT $(p<0,01)$, IP $(\mathrm{p}<0,01)$, and ILP $(\mathrm{p}<0,01) . \quad \mathrm{C}=$ Control; $\quad$ IC = Control Injury; ILT = Injury Low Level Laser Therapy; IP = Injury Platelet Rich Plasma; ILP = Injury LLLT and PRP.

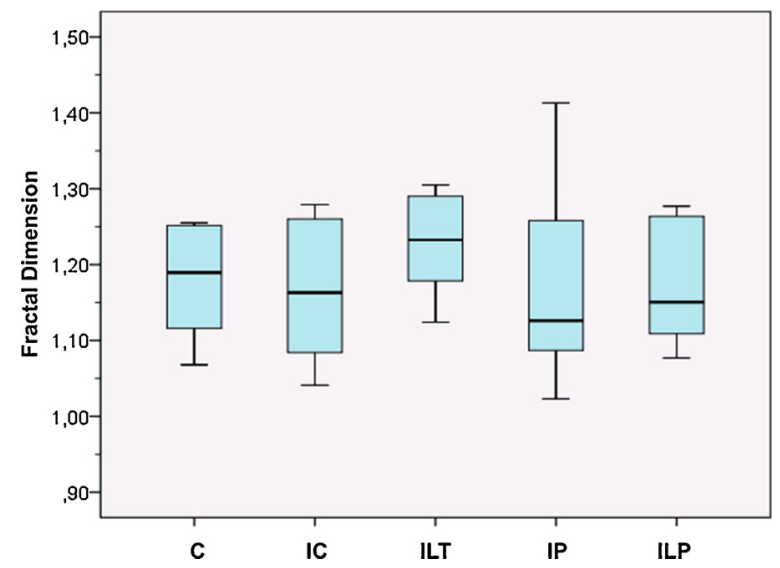

Fig. 5. Boxplot of fractal analysis of colagen in the slides stained with Picrosirius red and analysed by polarized light. $\mathrm{C}=\mathrm{Control}$; $\mathrm{IC}=\mathrm{Control}$ Injury; ILT = Injury Low Level Laser Therapy; IP = Injury Platelet Rich Plasma; ILP = Injury LLLT and PRP.

lymphocytes, macrophages, and polymorphonuclear cells occurs, making these phases more sensitive to the FD method (de Souza and Gottfried, 2013). In the present study, seven days after the injury, the muscles were in the repair phase, with intense inflammatory infiltrate and phagocytosis.

When observing the FD of the HE staining, the groups that suffered the injury process (IC, ILT, IP, ILP) presented higher values in relation to the control group (C). This fact can be verified in the qualitative analysis of the same slides in which the difference in group C compared to the other groups is clear, through the presence of inflammatory infiltrate on the slides.

The fact that the FD did not demonstrate differences between the treatment groups and the injury group, can be associated with the fact of that method is not sensitive to the nuclei on the images. The works using FD and gray level co-occurrence matrix (GLCM), for hippocampus, corpus callosum and cingulum analysis (Pantic et al., 2015, 2014), GLCM alone or associated with FD had better results than the FD method alone, this improvement was attributed to the ability of the technique to quantify the density of the observed areas. In our findings, the extent of the injury area was smaller in the treated groups, resulting in denser inflammatory infiltrate. Since FD is based on analysis of the nuclei, even with better histological aspects, the number of nuclei may have increased the FD, resembling the IC group.
After the destruction phase in which the muscle is infiltrated to clean the destroyed cells, the repair phase requires blood supply and polymorphonuclear cells that release growth factors for the regeneration and growth of new muscle cells. Low Level Laser Therapy includes factors that promote angiogenesis, in addition to having anti-inflammatory properties (Alves et al., 2014a; Alves et al., 2014b), while PRP increases the number of leukocytes at the injury site and promotes better tissue repair (Borrione et al., 2014; Li et al., 2013). These two factors were probably responsible for both the decrease in injury area and increase in the number of inflammatory cells in the infiltrate.

In a study by Cury et al. (2018) using the box counting method, the muscle disorganization of elderly rats with muscular dystrophy (mdx) and healthy animals was differentiated. The aspect of the dystrophic muscle resembles the injured muscle, showing affinity of the DF to differentiate between injured and healthy muscles. In addition, in the same study, a difference was found in the muscle collagen, which is thickened in the perimysium and epimisium, different from the muscle injured where the increase is focal at the spot of the lesion.

The perimysium and endomysium were evaluated by qualitative analysis demonstrated no alterations in any of the groups; however, the polarized collagen images exhibited a higher amount of collagen in the injured groups. This is due to the filling of the injury site by scar tissue, as seen in the literature (de Souza and Gottfried, 2013; Alves et al., 2014b). Another study that quantified collagen also did not find a significant difference by 7 days post injury (De Souza et al., 2011). This may indicate that the LLLT, previously shown (Luo et al., 2013) to decrease collagen production by inhibiting the production of TGF- $\beta 1$, was not sufficient to cause significant alterations. Within the composition of PRP, TGF- $\beta 1$ is also present (Cole et al., 2010), which could make PRP favorable for cicatricial fibrosis.

Fractal Dimension show us no differences between the control and the injured groups, one possibility to explain this could be that the collagen was more concentrated in the focal point of the injury rather than distributed between the fibers and fascicles as in group C. Neither treatment was able to significantly alter collagen production at 7 days after injury; however our study did not observe the injury until its resolution, where it would be possible to evaluate the cicatricial fibrosis.

In this way FD has been successfully used to identify cancer cells, discriminate the architecture of muscles with dystrophy as well as differentiate brain cells (Lee et al., 2014; Pantic et al., 2015, 2014; Cury et al., 2018), and these processes are linked to the number of nuclei in the analyzed site. In another study from our group (Ozaki et al., 2015), FD proved to be effective in differentiating injured muscle from healthy muscle, a fact that was again observed in the present study. Although, the box-counting method founded differences in FD of a healthy muscle to an injured in histological aspects, but it was not sensitive to differentiate two distinct treatments or to find differences in collagen levels.

The limitations of our study include the large withdrawal of blood for production of autologous PRP. This abrupt withdrawal of blood from the animal may impair its regeneration. The box-counting method used to evaluate Fractal Dimension captures and analyzes fewer objects in the image when compared to other methods like FracLac, making analysis less sensitive in more complex images.

In addition, the injury method used applies the same energy intensity to all animals regardless of the weight or size of the animal.

\section{Conclusion}

We concluded that both treatments were able to accelerate repair of muscle injury and the association of both was better than application of each treatment in isolation. However, the method used to measure the FD was not sensitive to detect differences between treatments in neither the histological aspects of the injury nor the collagen. 

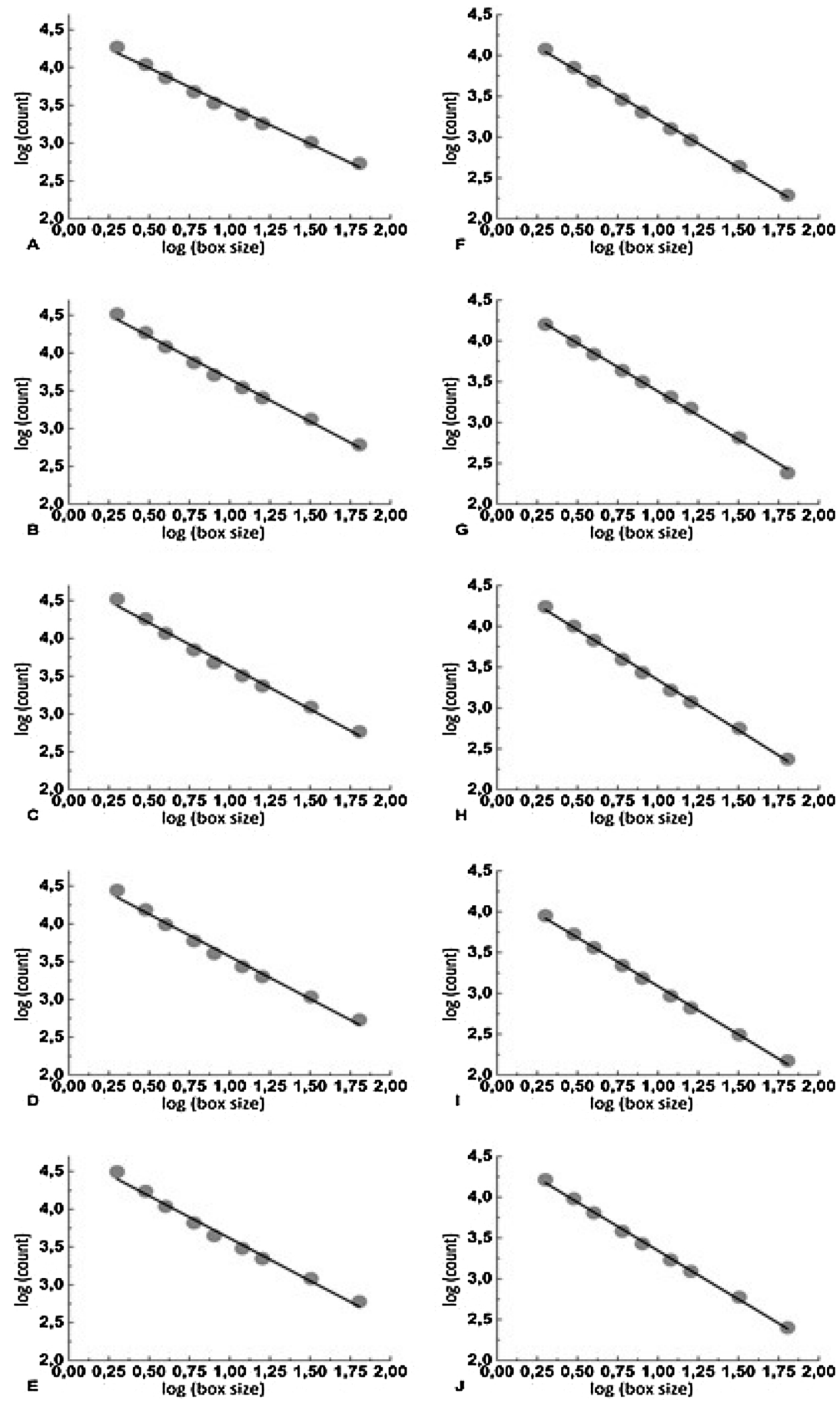

Fig. 6. Plots $\log \log$ for each group analysed. A = Control HE; B = Control Injury HE; C = Injury Low Level Laser Therapy HE; D = Injury Platelet Rich Plasma HE; $\mathrm{E}=$ Injury LLLT and PRP HE; F = Control colagen; G = Control Injury colagen; H = Injury Low Level Laser Therapy colagen; I = Injury Platelet Rich Plasma colagen; $\mathrm{J}=$ Injury LLLT and PRP colagen. 


\section{Funding}

This study was financed in part by the Coordenação de aperfeiçoamento de Pessoal de Nível Superior - Brasil (CAPES) - Finance Code 001

\section{Conflicts of interest}

The authors declare no conflicts of interest.

\section{References}

Adabbo, M., Paolillo, F.R., Bossini, P.S., Rodrigues, N.C., Bagnato, V.S., Parizotto, N.A. 2016. Effects of low-level laser therapy applied before treadmill training on recovery of injured skeletal muscle in wistar rats. Photomed. Laser Surg. [Internet] 34 (5), 187-193. Available from: http://online.liebertpub.com/doi/10.1089/pho.2015. 4031.

Alves, A.N., Fernandes, K.P.S., Deana, A.M., Bussadori, S.K., Mesquita-Ferrari, R.A., 2014a. Effects of low-level laser therapy on skeletal muscle repair. Am J. Phys. Med. Rehabil. [Internet] 93 (12), 1073-1085. Available from: http://content.wkhealth. com/linkback/openurl?sid = WKPTLP:landingpage $\& a n=00002060-201412000$ 00007.

Alves, A.N., Fernandes, K.P.S., Melo, C.A.V., Yamaguchi, R.Y., França, C.M., Teixeira, D.F., et al., 2014b. Modulating effect of low level-laser therapy on fibrosis in the repair process of the tibialis anterior muscle in rats. Lasers Med. Sci. 29 (2), 813-821.

Astur, D.C., Novaretti, J.V., Uehbe, R.K., Arliani, G.G., Moraes, E.R., de Castro Pochini, A., et al., 2014. Muscle injury: current perspectives and trends in Brazil. Rev. Bras. Ortop. (English Ed [Internet] 49 (6), 573-580. Available from: http://linkinghub.elsevier. $\mathrm{com} /$ retrieve/pii/S2255497114001670.

Borrione, P., Grasso, L., Chierto, E., Geuna, S., Racca, S., Abbadessa, G., et al., 2014. Experimental model for the study of the effects of platelet-rich plasma on the early phases of muscle healing. Blood Transfus [Internet]. 12 (Suppl 1), s221-s228. Available from: http://www.pubmedcentral.nih.gov/articlerender.fcgi?artid= 3934271\&tool $=$ pmcentrez\&rendertype $=$ abstract.

Castoldi, R.C., Camargo, R.C.T., Magalhães, A.J.B., Ozaki, G.A.T., Kodama, F.Y., Oikawa, S.M., et al., 2013. Concurrent training effect on muscle fibers in Wistar rats. Mot. Rev. Educ Física [Internet]. 19 (December 4), 717-723. Available from: http://www. scielo.br/scielo.php?script $=$ sci_arttext\&pid $=$ S1980-65742013000400008\&lng $=$ en \&nrm $=$ iso\&tlng $=$ en.

Cole, B.J., Seroyer, S.T., Filardo, G., Bajaj, S., Fortier, L.A., 2010. Platelet-rich plasma: where are we now and where are we going? Sports Health 2 (3), 203-210.

Cury, S.S., Freire, P.P., Martinucci, B., dos Santos, V.C., de Oliveira, G., Ferretti, R., et al., 2018. Fractal dimension analysis reveals skeletal muscle disorganization in $\mathrm{mdx}$ mice. Biochem. Biophys. Res. Commun. 1-7.

de Melo de Mendonça, M.B., de Amorim, Garcia, C.A., de Albuquerque Nogueira, R., Andreade Filgueira Gomes, M., Morais Valença, M., Oréfice, F., 2007. Análise fractal da vasculatura retínica: Métodos de segmentação e de cálculo dimensional. Arq. Bras. Oftalmol. 70 (3), 413-422.

de Souza, J., Gottfried, C., 2013. Muscle injury: review of experimental mo dels. J Electromyogr Kinesiol [Internet] 23 (6), 1253-1260. https://doi.org/10.1016/j. jelekin.2013.07.009. Available from:.

De Souza, T.O.F., Mesquita, D.A., Ferrari, R.A.M., Dos Santos Pinto, D., Correa, L. Bussadori, S.K., et al., 2011. Phototherapy with low-level laser affects the remodeling of types i and III collagen in skeletal muscle repair. Lasers Med. Sci. 26 (6), 803-814.

Dimauro, I., Grasso, L., Fittipaldi, S., Fantini, C., Mercatelli, N., Racca, S., et al., 2014 Platelet-rich plasma and skeletal muscle healing: a molecular analysis of the early phases of the regeneration process in an experimental animal model. PLoS One 9 (7).

Edouard, P., Branco, P., Alonso, J.-M., 2016. Muscle injuries and hamstring muscle injuries during the international athletics championships between 2007 and 2015. Ann.
Phys. Rehabil. Med. 59 (September), e22.

Li, H., Usas, A., Poddar, M., Chen, C., Thompson, S., Ahani, B., et al., 2013. Platelet-Rich plasma promotes the proliferation of human muscle derived progenitor cells and maintains their stemness. Prosper F, editor. PLoS One [Internet] 8 (6), e64923. June 7. http://dx.plos.org/10.1371/journal.pone.0064923.

Gupta, S., Hartley, R., Khan, U.T., Singapuri, A., Hargadon, B., Monteiro, W., et al., 2014 Quantitative computed tomography-derived clusters: redefining airway remodeling in asthmatic patients. J. Allergy Clin. Immunol. [Internet] 133 (3), 729-738. https:// doi.org/10.1016/j.jaci.2013.09.039. e18. Available from:

Hammond, J.W., Hinton, R.Y., Curl, L.A., Muriel, J.M., Lovering, R.M., 2009. Use of autologous platelet-rich plasma to treat muscle strain injuries. Am. J Sports Med. [Internet] 37 (6), 1135-1142. https://doi.org/10.1177/0363546508330974. Available from:

Iyomasa, D.M., Garavelo, I., Iyomasa, M.M., Watanabe, I., Issa, J.P.M., 2009. Ultrastructural analysis of the low level laser therapy effects on the lesioned anterior tibial muscle in the Gerbil. Micron 40 (June 4), 413-418.

JCSC, Filho, Garcia, B.C., Kodama, F.Y., Bonfim, M.R., Vanderlei, L.C.M., Ramos, E.M.C., et al., 2011. Efeitos do exercício aeróbio no músculo esquelético de ratos expostos à fumaça de cigarro. Rev. Bras. Med. do Esporte. 17 (6), 416-419.

Lee, L.H., Tambasco, M., Otsuka, S., Wright, A., Klimowicz, A., Petrillo, S., et al., 2014. Digital differentiation of non-small cell carcinomas of the lung by the fractal dimension of their epithelial architecture. Micron [Internet] 67, 125-131. https://doi org/10.1016/j.micron.2014.07.005. Available from:.

Li, W., Enomoto, M., Ukegawa, M., Hirai, T., Sotome, S., Wakabayashi, Y., et al., 2012. Subcutaneous injections of platelet-rich plasma into skin flaps modulate proangiogenic gene expression and improve survival rates. Plast. Reconstr. Surg. [Internet] 129 (4), 858-866. Available from: http://content.wkhealth.com/linkback/openurl? sid = WKPTLP:landingpage\&an =00006534-201204000-00014.

Li, H., Hicks, J.J., Wang, L., Oyster, N., Philippon, M.J., Hurwitz, S., et al., 2016. Customized platelet-rich plasma with transforming growth factor $\beta 1$ neutralization antibody to reduce fibrosis in skeletal muscle. Biomaterials [Internet] 87 (May), 147-156. Available from: http://linkinghub.elsevier.com/retrieve/pii/ S0142961216001319.

Luo, L., Sun, Z., Zhang, L., Li, X., Dong, Y., Liu, T.C.Y., 2013. Effects of low-level laser therapy on ROS homeostasis and expression of IGF-1 and TGF- $\beta 1$ in skeletal muscle during the repair process. Lasers Med. Sci. 28 (3), 725-734.

Moreira, R.D., Moriel, A.R., Murta Junior, L.O., Neves, L.A., Godoy, M.F., 2011. Fractal dimension in quantifying the degree of myocardial cellular rejection after cardiac transplantation. Rev. Bras. Cir Cardiovasc Orgao Of da Soc Bras Cir Cardiovasc. 26 (2), 155-163.

Ozaki, G.A.T., Camargo, R.C.T., Koike, T.E., Garcia, T.A., Castoldi, R.C., Camargo Filho, J.C.S., 2015. Fractal analysis of skeletal muscle tissue of rats subjected to stretch injury. Int. J. Morphol. 33 (3).

Pachioni, C.A.S., Mazzer, N., Barbieri, C.H., de Camargo, M.R., Fregonesi, C.E.P.T., do Carmo, E.M., et al., 2009. Stretch injuries of skeletal muscles: experimental study in rats' soleus muscle. Int. J. Morphol. [Internet] 27 (4), 1139-1146. Available from: http://www.scielo.cl/scielo.php?script $=$ sci_arttext\&pid $=$ S0717 95022009000400029\&lng $=$ en\&nrm $=$ iso\&tlng $=$ en

Pantic, I., Dacic, S., Brkic, P., Lavrnja, I., Pantic, S., Jovanovic, T., et al., 2014. Application of fractal and grey level co-occurrence matrix analysis in evaluation of brain corpus callosum and cingulum architecture. Microsc. Microanal. 20 (5), 1373-1381.

Pantic, I., Dacic, S., Brkic, P., Lavrnja, I., Jovanovic, T., Pantic, S., et al., 2015. Discriminatory ability of fractal and grey level co-occurrence matrix methods in structural analysis of hippocampus layers. J. Theor. Biol. [Internet] 370, 151-156. https://doi.org/10.1016/j.jtbi.2015.01.035. Available from:

Ristanović, D., Stefanović, B.D., Puškaš, N., 2014. Fractal analysis of dendrite morphology using modified box-counting method. Neurosci. Res. 84 (July), 64-67.

Silveira, P.C.L., Scheffer D da, L., Glaser, V., Remor, A.P., Pinho, R.A., Aguiar Junior, A.S., et al., 2016. Low-level laser therapy attenuates the acute inflammatory response induced by muscle traumatic injury. Free Radic. Res. [Internet] 50 (5), 503-513. Available from: http://www.tandfonline.com/doi/full/10.3109/10715762.2016. 1147649. 